\title{
SNR Improvement by Photon Noise Filtering in Ocean Color Monitor Satellite Images
}

\author{
Ashok Kumar ${ }^{1}$, Rajiv Kumaran \\ Space Applications Centre, Indian Space Research Organization, Ahmedabad, India \\ Email: ashokkumar@sac.isro.gov.in \\ Harsh C Trivedi ${ }^{2}$ \\ L D College of Engineering, Ahmedabad, India \\ Email: hctrivedi3@gmail.com
}

\begin{abstract}
In high radiometric resolution electro optical image payloads of remote sensing satellites, photon noise dominates SNR performance. Photon noise is input signal dependent and difficult to filter. This paper proposes a photon noise filtering technique for Ocean Color Monitor (OCM) images. Existing filtering techniques are meant for object detection and handles images with poor SNR. As OCM SNR is on higher side, custom sigma filter based denoising technique is developed. Proposed technique first converts photon noise to signal independent Gaussian noise. For this variance stabilization, Anscombe transform is used. Simulations are carried on various images. Proposed technique provides $20-50 \%$ reduction in overall as well count-wise RMSE. FFT analysis shows significant reduction in noise. Proposed technique is of low complexity.
\end{abstract}

Index Terms - Photon Noise, Signal to Noise Ratio, Ocean Color Monitor, Sigma Filter, Variance stabilization, Root Mean Square Error.

\section{INTRODUCTION}

ISRO's Oceansat-2 satellite carried three payloads, Ocean Color Monitor (OCM), Ku-band Pencil Beam Scatterometer and Radio Occultation Sounder for Atmosphere (ROSA). The payloads of this mission are tailored for making measurements of the physical and the biological oceanographic parameters.

OCM-2 has eight multi-spectral bands in VNIR region. With IGFOV (Instantaneous Geometric Field of View) of $360 X 236 \mathrm{~m}$ it covers swath of $1420 \mathrm{Km}$. At sea reference radiance, it provides PSNR $\sim 300$ with 12-bit digitization.

In any electro-optical imaging system, SNR (Signal to Noise Ratio) defines quality of measurement within detection range [1]. Definition of SNR is as follows.

$$
\mathrm{SNR}_{\text {Radiance }}=\frac{\text { Signal }_{\text {Radiance }}}{\text { Noise }_{\text {Radiance }}}
$$

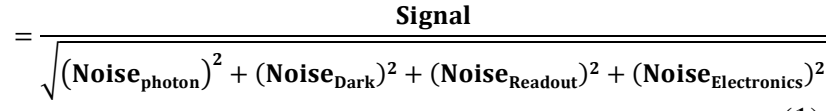

In OCM-2, system SNR performance is mainly limited by photon noise, which follows Poisson distribution.

$$
P(y \mid x)=\frac{x^{y}}{y !} e^{-x}
$$

The mean and variance of a Poisson process are equal to the intensity. The intensity/mean is the "signal" of interest and the variability of the data about the mean can be interpreted as "noise." Thus, as the intensity varies spatially as a function of astrophysical structure and function, so does the signal-to-noise ratio. In this sense it could be said that the noise in photon-limited imaging is signal-dependent.

For photon noise reduction, literature suggests few denoising methods like Non-local PCA (Principle Component Analysis) transform and sparse transform [25], but these techniques are mainly meant for additive Gaussian noise and provide improvement for images with poor SNR only $(<40 \mathrm{~dB})$. These techniques helps in object detection. These techniques don't improve radiometric performance. In OCM case, SNR is on higher side. For images with high SNR, variance stabilizing transforms (VST) such as the Anscombe VST [6, 7] or Fisz Transform [8, 9] offer a pragmatic solution for Poisson noise removal. The VST stabilizes the variance of the Poisson image to a constant, and the resulting image tends to homo-skedastic Gaussian as the intensity of the original image tends to infinity. VST by Anscombe is a simpler solution.

$$
f_{\text {Anscombe }}(x)=2 \sqrt{x+\frac{3}{8}}
$$

For this specifically converted Gaussian noise removal, among many algorithms, which exist in the open literature, the sigma filter [10] is probably one of the simplest de-noising methods. Due to its simplicity, this filter represents a good choice for implementation. 
However, the edge preservation performance of the sigma filter is not good, especially for small image details with variance close to the variance of the additive noise. In order to improve the detail preservation of the sigma filter, other more sophisticated approaches are proposed. For instance, proposed fuzzy filter [11] uses some fuzzy estimates of the local derivative to perform directional filtering of the image. Although it provides good filtering performances, this approach have the disadvantage of relative high complexity. Another alternative, hybrid sigma filter [12] is also proposed for speckle noise reduction. The hybrid sigma filter, however, does not address the problem of additive noise reduction which is the focus of our work.

This paper provides a new photon noise filtering which combines Anscombe transformation [6] and modified Sigma filter [13] for photon noise reduction in images. In our proposed method the input image is first decomposed in four components and a sigma filter is applied separately on each of them. The output image is then reconstructed from the four filtered components.

The paper is organized as follows. In section 2 , the modified sigma filter is described. In section 3 , the denoising algorithm is summarized. Section 4 provides results.

\section{MODIFIED SIGMA FILTER}

First, we briefly review the standard sigma filter for additive noise [10] and outline its advantages and disadvantages. We assume the following model for the input image:

$$
y(i, j)=x(i, j)+n(i, j)
$$

' $y(i, j)$ ' is the observed image, ' $x(i, j)$ ' is the original clean image and ' $n(i, j)$ ' is a zero mean Gaussian distributed additive noise.

The main idea of the sigma filter is based on the fact that for a Gaussian distributed variable with mean $\mu$ and variance $\sigma^{2}$, a percentage of $95.5 \%$ of its samples lies inside the range $[\mu-2 \sigma, \mu+2 \sigma]$. Applying this observation to the model from (4), for every pixel $y(i, j)$ from the observed image, a local average is computed on those neighboring pixels (in window of $\mathbf{M} \times \mathbf{M}$ ) that are inside the interval $[y(i, j)-2 \sigma, y(i, j)+2 \sigma]$. Corresponding pixel of the output image $f(i, j)$ is replaced with this local average. A block diagram for de-noising based on sigma filter is depicted in Fig. 1.

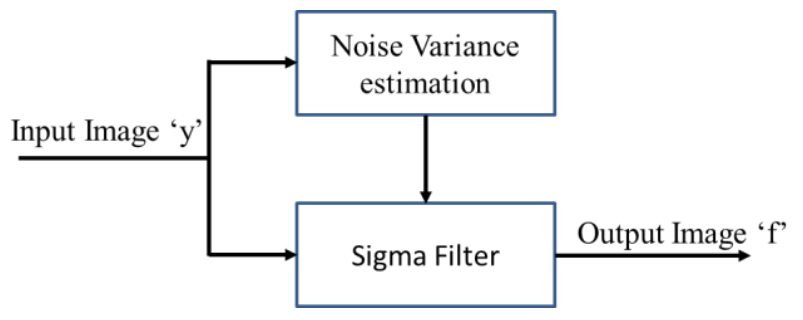

Fig.1. Denoising based on Sigma Filter
The input image is first passed through the noise estimation module and the estimated noise variance is then used in the sigma filter for de-noising. It is well known, that small details of the input image are not well preserved by the sigma filter. This is due to the fact that on the regions from the input image that have variance close to the noise variance almost all pixels from the local $\mathrm{M} \times \mathrm{M}$ window are used in the average process. For instance when the estimated noise variance is larger than the real noise level the blurring effect is evident. An immediate solution is to decrease the length of the selection range to $[\mathrm{y}(\mathrm{i}, \mathrm{j})-\mathrm{k} \sigma, \mathrm{y}(\mathrm{i}, \mathrm{j})+\mathrm{k} \sigma]$ with $\mathrm{k}<2$. This modification reduces also the filtering capabilities of the sigma filter in smooth areas of the input image.

Taking into account these observations, we introduce a simple modification that improves the performances of the sigma filter for regions of the input image that contain small details.

The block diagram of our proposed Sigma filter is depicted in Fig. 2, where the blocks denoted as HPV and HPH perform a high-pass filtering on the vertical and respectively horizontal directions. Noise estimation is performed by the blocks denoted as NE and the sigma filters are represented by the blocks Sigma.

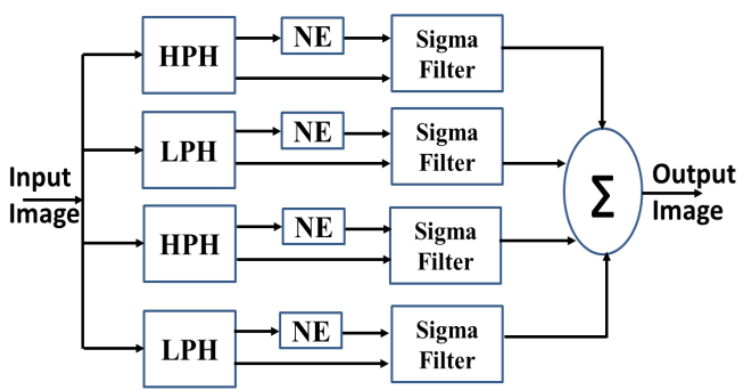

Fig.2. Proposed Modified Sigma Filter based denoising technique

Four component decomposition can be performed as follows:

$$
\begin{aligned}
& Y_{L P H}(i, j)=[I(i, j)+I(i, j+1)] / 2 \\
& Y_{H P H}(i, j)=[I(i, j)-I(i, j+1)] / 2 \\
& Y_{L P V}(i, j)=[I(i, j)+I(i+1, j)] / 2 \\
& Y_{H P V}(i, j)=[I(i, j)-I(i+1, j)] / 2
\end{aligned}
$$

Computation of these differences transforms the horizontal monotonically increasing/decreasing regions of the input image into constant regions [14]. Moreover this operation also preserves the edges from the input image. Transformation of the monotonic regions into constant regions makes the simple averaging, performed by the sigma filter, a better model. The coefficient $1 / 2$ is introduced to preserve the dynamic range. It does not influence the filtering accuracy and it can be discarded at this point.

If $\mathrm{Y}(\mathrm{i}, \mathrm{j})$ has noise variance $\sigma_{\mathrm{n}}{ }^{2}$ then noise variance of four components can be written as [r14]: 


$$
\sigma_{L P H}^{2}=\sigma_{H P H}^{2}=\sigma_{L P V}^{2}=\sigma_{H P V}^{2}=\frac{\sigma_{n}^{2}}{2}
$$

Reverse computation can be done by:

$$
\begin{array}{r}
\mathrm{Y}(\mathrm{i}, \mathrm{j})=\left[\mathrm{Y}_{\mathrm{LPH}}(\mathrm{i}, \mathrm{j})+\mathrm{Y}_{\mathrm{HPH}}(\mathrm{i}, \mathrm{j})+\mathrm{Y}_{\mathrm{LPV}}(\mathrm{i}, \mathrm{j})+\mathrm{Y}_{\mathrm{HPV}}\right. \\
(\mathrm{i}, \mathrm{j})] / 2
\end{array}
$$

\section{Proposed DenOIsING Algorithm}

Typical flow chart of proposed denoising algorithm is given below.

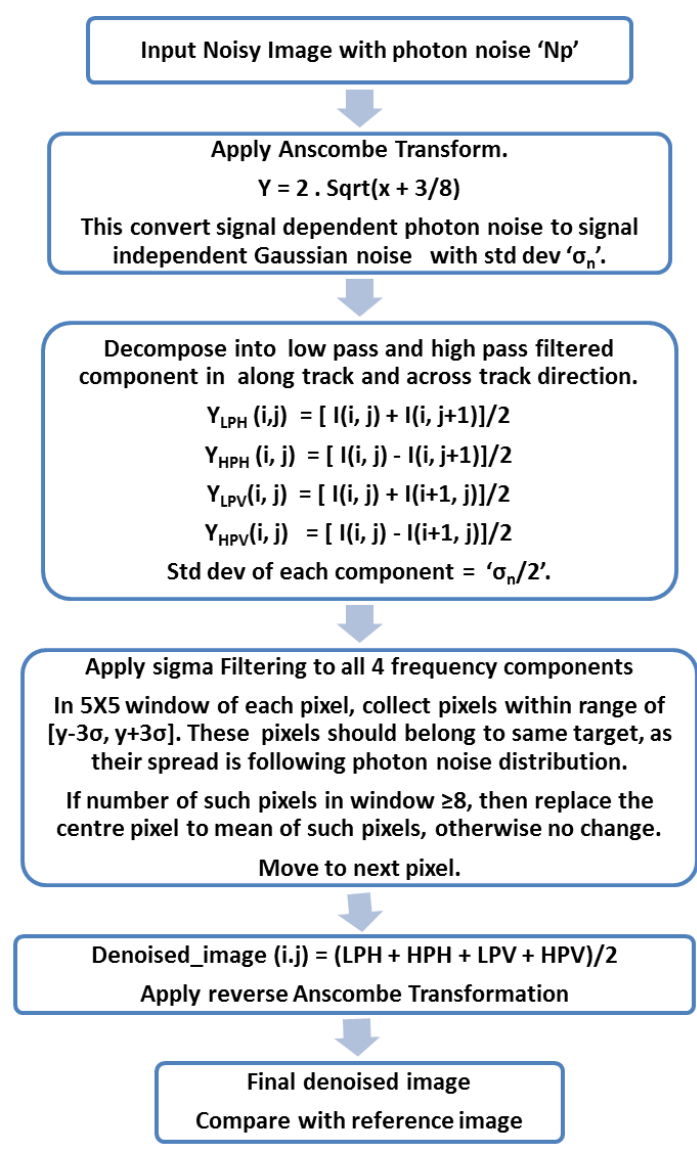

\section{SIMULATION IMAGE-SETS}

As no ground truth data is available, radio-metrically corrected 12-bit OCM-B1 images with PSNR=1000 is considered as reference image-set. Pixel wise Poisson distributed noise (with PSNR $=256$ ) is added to this image and noise filter (as mentioned in previous section) is applied. Filtered image is compared with reference image to evaluated denoising performance.

Fig. 3-7 shows these image sets. Respective histograms are shown in Fig. 8-12. Table 1 shows parameters of simulation image-sets. For these parameters, equations are shown below [15].

$$
\text { Mean }=\frac{1}{m} \cdot \frac{1}{n} \sum_{i=0}^{m-1} \sum_{j=0}^{n-1} \operatorname{Im} \operatorname{age}(i, j)
$$

$$
S t d-\operatorname{dev}=\sqrt{\frac{1}{m} \cdot \frac{1}{n} \sum_{i=0}^{m-1} \sum_{j=0}^{n-1}[\operatorname{Im} \operatorname{age}(i, j)-\text { Mean }]^{2}}
$$

$$
\text { Line_complexity }=\frac{1}{m} \cdot \frac{1}{n-1} \sum_{i=0}^{m-1} \sum_{j=0}^{n-2}|\operatorname{Im} \operatorname{age}(i, j)-\operatorname{Im} \operatorname{Ige}(i, j+1)|
$$

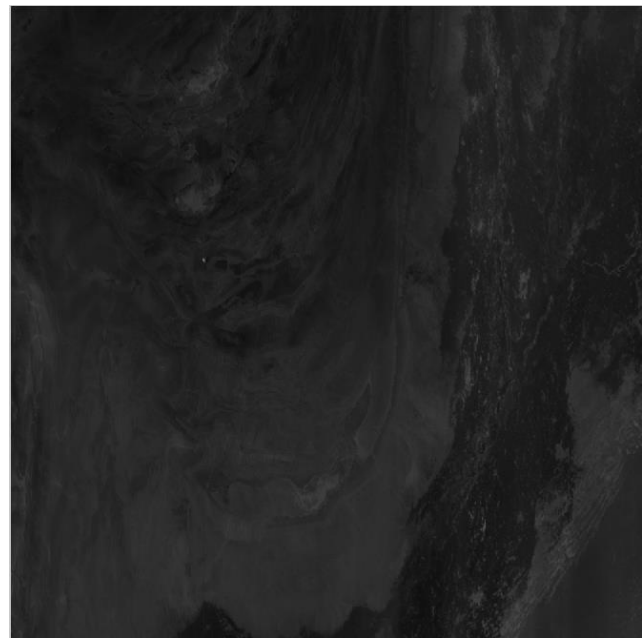

Fig.3. Simulation Image-set 1 (1000X1000)

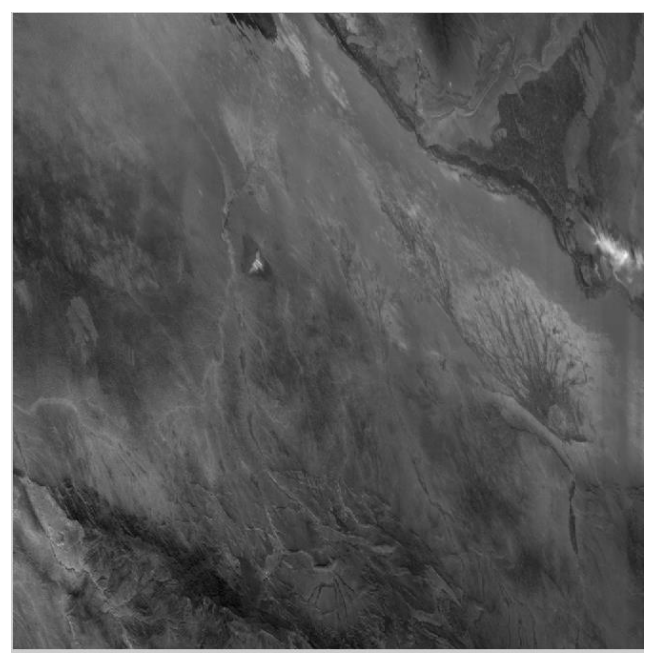

Fig.4. Simulation Image-set 2 (1000X1000)

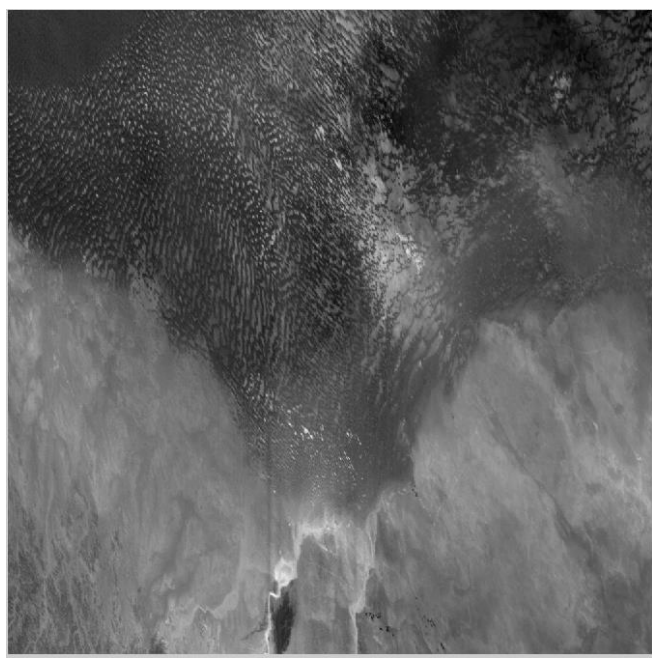

Fig.5. Simulation Image-set 3 (1000X1000) 


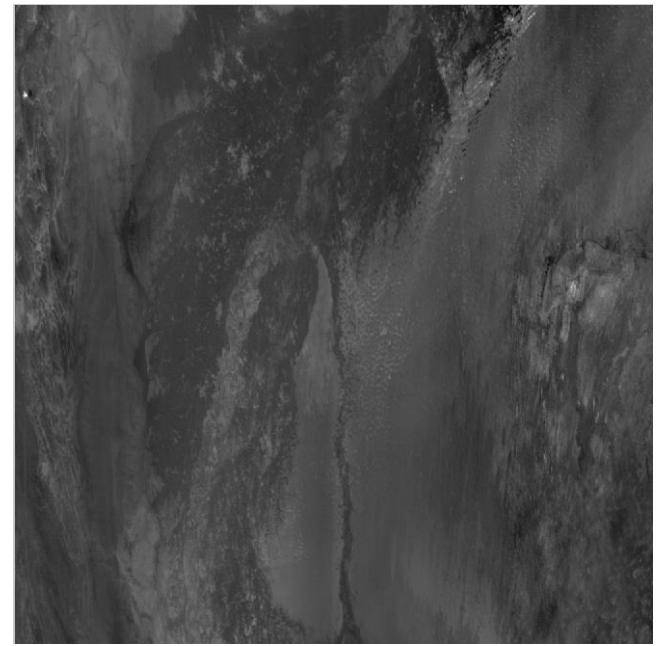

Fig.6. Simulation Image-set 4 (1000X1000)

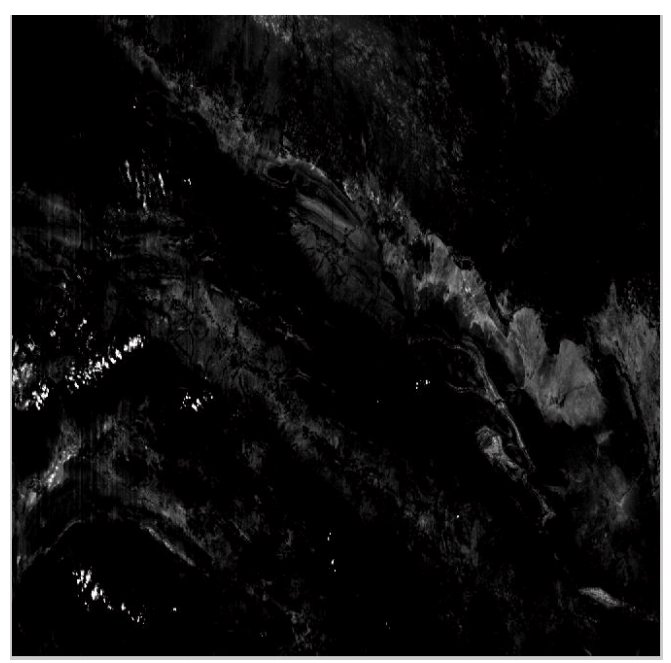

Fig.7. Simulation Image-set 5 (1000X1000)

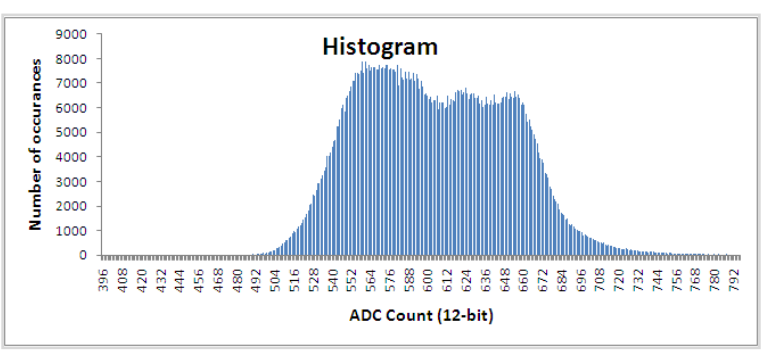

Fig.8. Histogram of Simulation Image-set 1

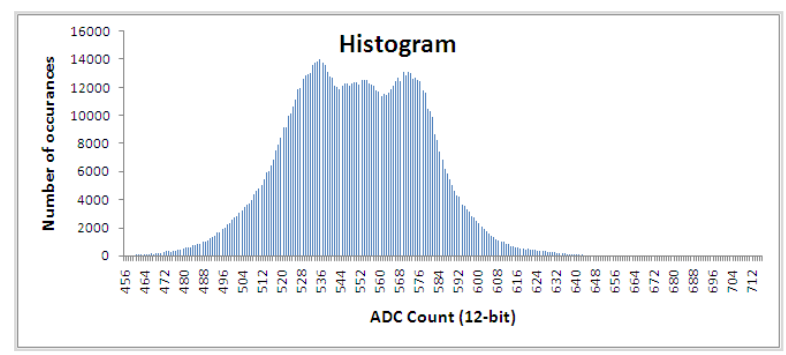

Fig.9. Histogram of Simulation Image-set 2

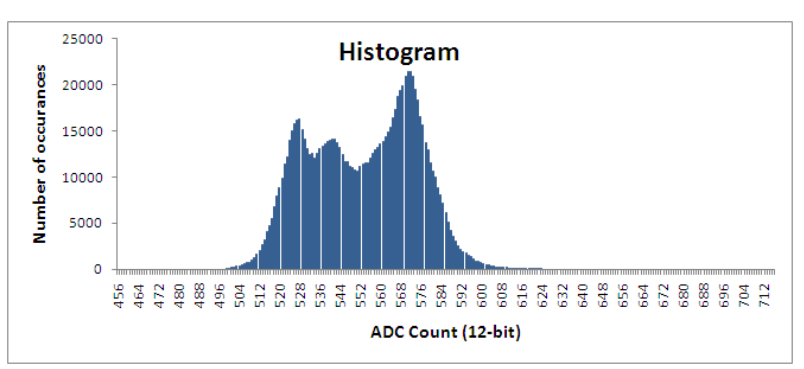

Fig.10. Histogram of Simulation Image-set 3

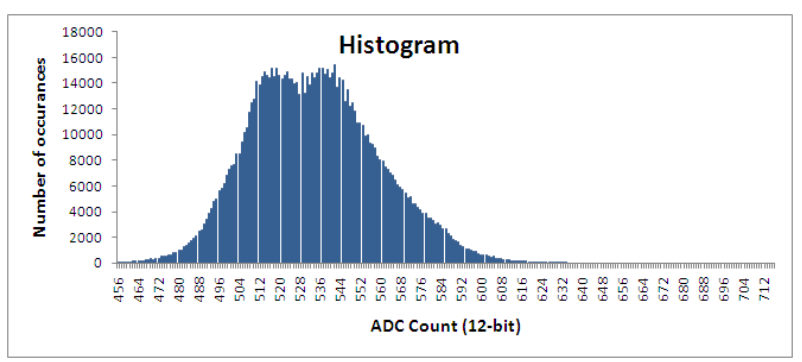

Fig.11. Histogram of Simulation Image-set 4

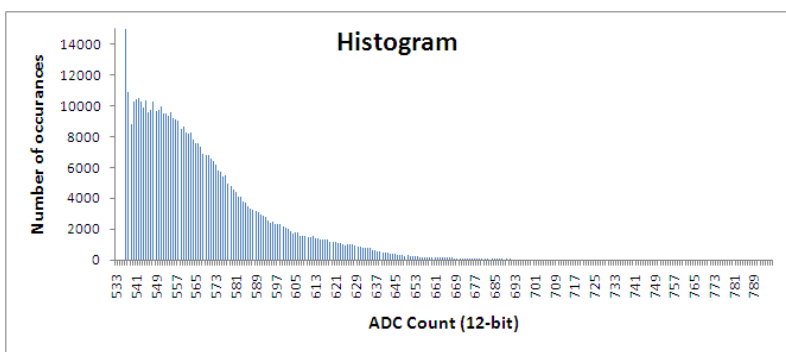

Fig.12. Histogram of Simulation Image-set 5

Table 1. Image parameters of Simulation image-sets

\begin{tabular}{|c|c|c|c|c|}
\hline & $\begin{array}{l}\text { Image- } \\
\text { size }\end{array}$ & Mean & Std-dev & Row complexity \\
\hline Image-1 & \multirow{5}{*}{ 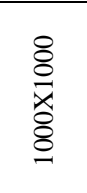 } & 604 & 46 & 8 \\
\hline Image-2 & & 550 & 27 & 4.4 \\
\hline Image-3 & & 553 & 21 & 3.5 \\
\hline Image-4 & & 554 & 25 & 6.2 \\
\hline Image-5 & & 552 & 26 & 4.9 \\
\hline
\end{tabular}

\section{SimULATION RESUlTS}

Noisy images with PSNR $=250$ are filtered by denoising approach explained in previous sections. This section highlights results. Table-2 provide RMSE results comparison between noisy and denoised image.

Table 2. RMSE results with noisy images of PSNR $=250$

\begin{tabular}{|c|c|c|c|}
\hline & $\begin{array}{c}\text { RMSE } \\
\text { Noisy Image }\end{array}$ & $\begin{array}{c}\text { RMSE } \\
\text { Denoised Image }\end{array}$ & $\begin{array}{c}\text { Mismatch (\%) } \\
\text { Denoised }\end{array}$ \\
\hline Image-1 & 6.3 & 4.6 & 0.83 \\
\hline Image-2 & 6.0 & 3.6 & 0.87 \\
\hline Image-3 & 6.0 & 3.1 & 0.87 \\
\hline Image-4 & 6.9 & 4.1 & 0.88 \\
\hline Image-5 & 6.0 & 3.6 & 0.87 \\
\hline
\end{tabular}


For computing SNR at every count ' $\mathrm{S}$ ', following equation is used.

$$
\begin{aligned}
& \operatorname{SNR}(S)=20 \log _{10} \\
& {\left[\frac{S}{\sqrt{\sum_{i, j\left(\text { ref_image }(i, j) \in \epsilon^{\prime} S^{\prime}\right)}\left([S-\operatorname{denoised}(i, j)]^{2}+\left[\text { Noise }_{\text {system }}(S)\right]^{2}\right)}} \cdot \operatorname{hist}(S)\right]}
\end{aligned}
$$

Figure 13-17 provide SNR improvement analysis [15] in Image-set1 to 5. Lesser SNR improvement is also because of less number of occurrence of that particular count (refer histogram in Fig 8-12). This may be noted that proposed algorithm provides more improvement with images of lower contrast.

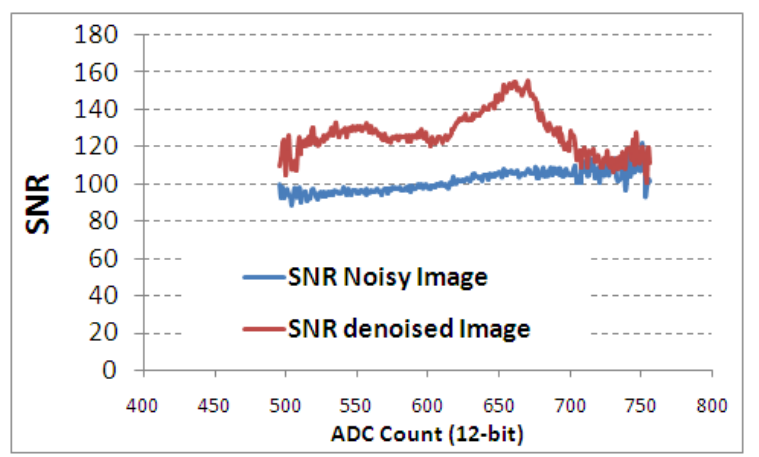

Fig.13. SNR improvement in Image-set1

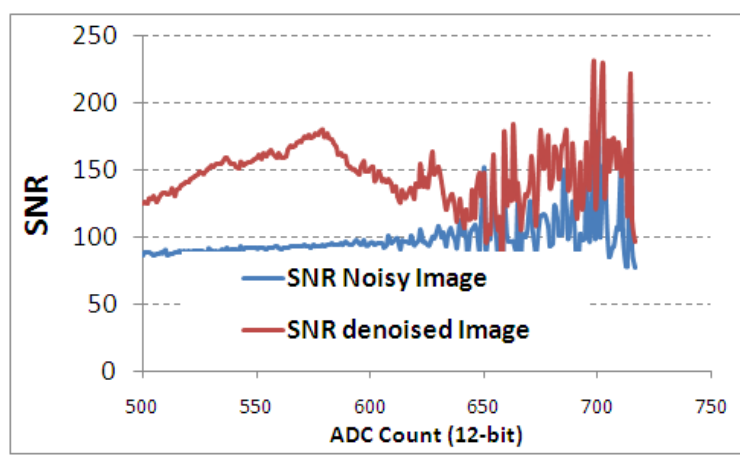

Fig.14. SNR improvement in Image-set2

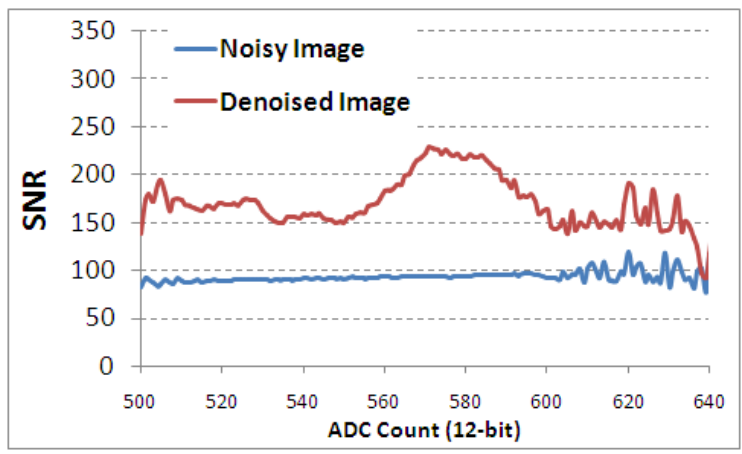

Fig.15. SNR improvement in Image-set3

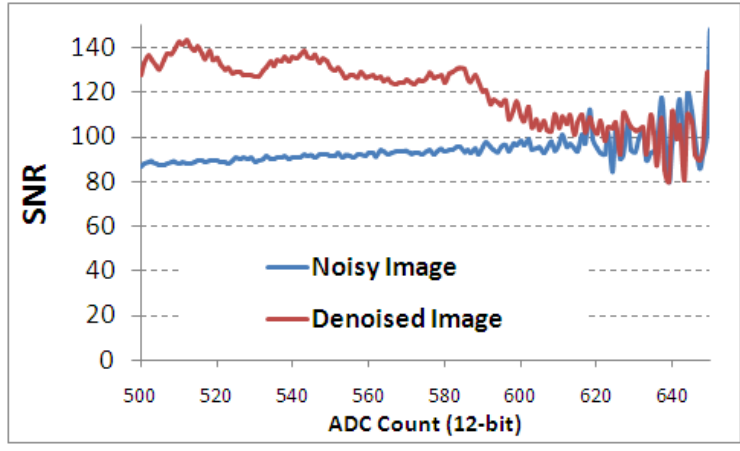

Fig.16. SNR improvement in Image-set4

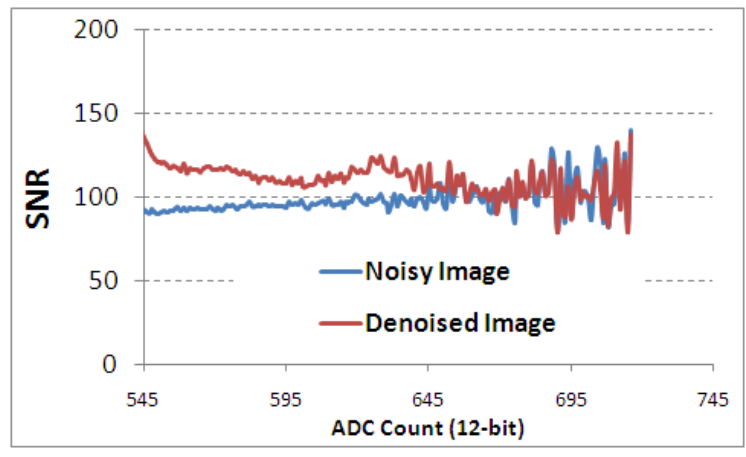

Fig.17. SNR improvement in Image-set5

For Image-set1 \& 2, RMSE comparison analysis is carried out count wise and shown in Fig. $18 \& 19$.

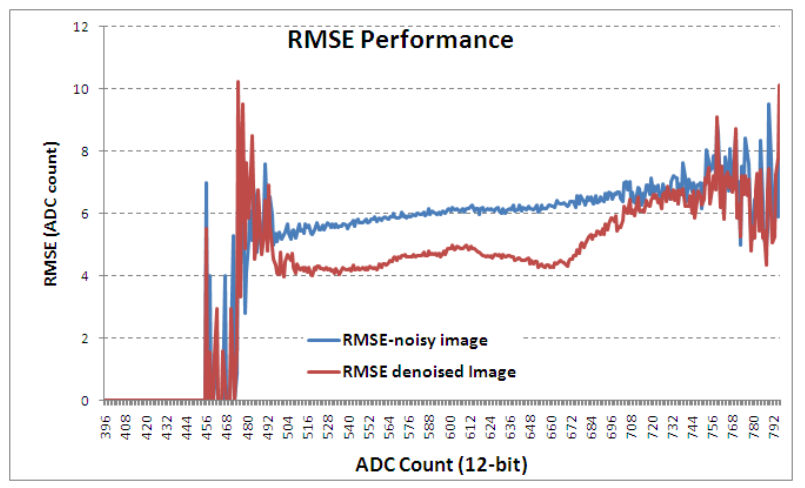

Fig.18. RMSE analysis of Image-set1

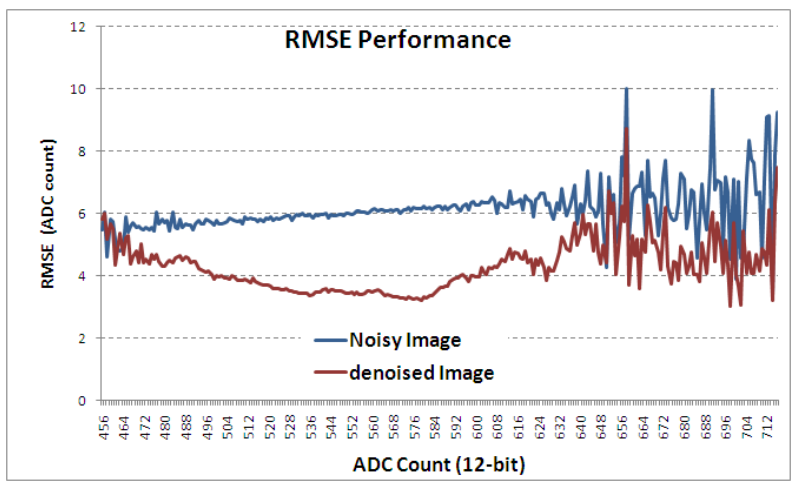

Fig.19. RMSE analysis of Image-set2 
For Image-set1 \& 2, peak to peak error comparison analysis is carried out count wise and shown in Fig. 20. Proposed algorithm does not increases peak to peak error.

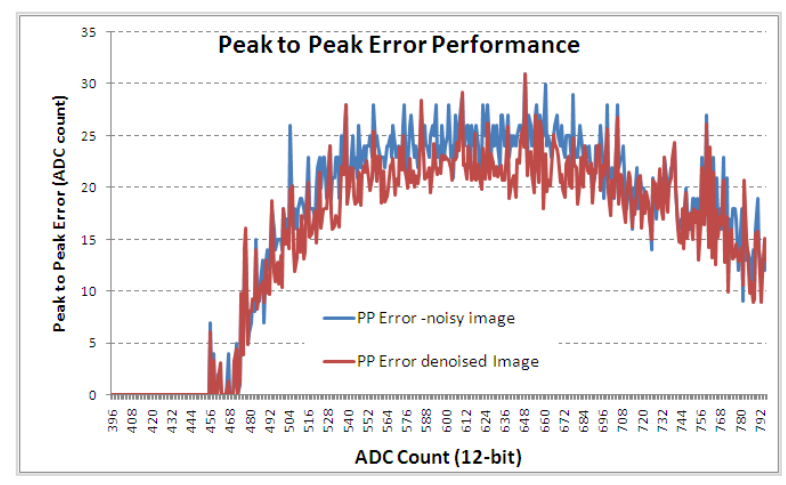

Fig.20. Peak to Peak error analysis of Image-set1

FFT Analysis is also carried out on reference image, noisy image and denoised image. For image-set1, FFT analysis of along-track data is shown is shown in Fig. 21 $\& 22$. It can be assumed that natural ocean data will be of low frequency only. High frequency content will be due to noise only. With filtering, high frequency noise reduces significantly. FFT analysis of across track data is shown in Fig. 23.

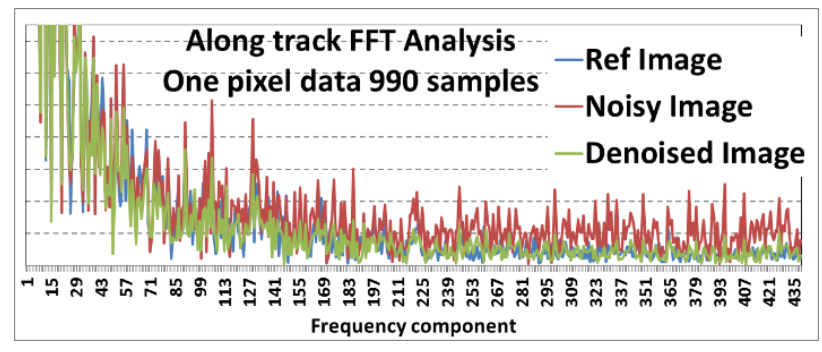

Fig.21. FFT Analysis for along track data in Image-set1

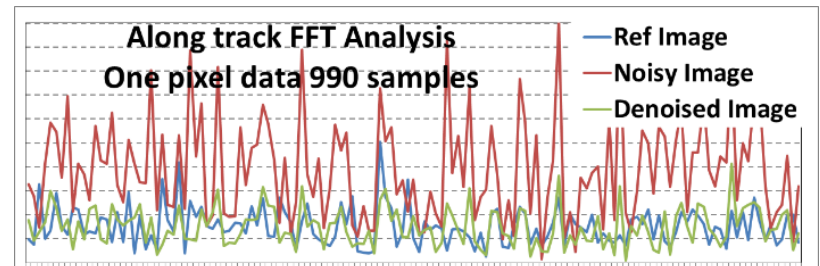

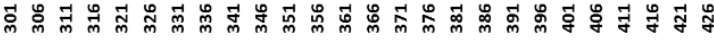
Frequency component

Fig.22. FFT Analysis for along track data in Image-set1 (zoomed form of Fig. 21)

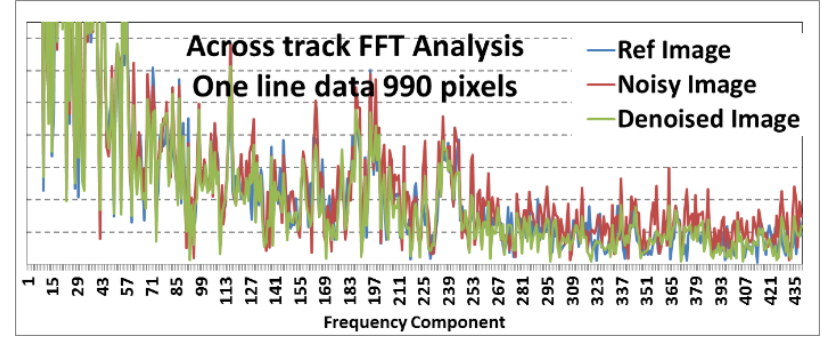

Fig.23. FFT Analysis for across track data in Image-set1
Similar to noisy images with PSNR 250, two more image-sets with PSNR of $125 \& 500$ are generated. RMSE simulation results with these images are shown in Table 3 \& 4 .

Table 3. RMSE results with noisy images of PSNR $=125$

\begin{tabular}{|c|c|c|}
\hline & $\begin{array}{c}\text { RMSE } \\
\text { Noisy Image }\end{array}$ & $\begin{array}{c}\text { RMSE } \\
\text { Denoised Image }\end{array}$ \\
\hline Image-1 & $\mathbf{1 2 . 6}$ & $\mathbf{1 0 . 0}$ \\
\hline Image-2 & $\mathbf{1 2 . 0}$ & $\mathbf{8 . 9}$ \\
\hline Image-3 & $\mathbf{1 2 . 0}$ & $\mathbf{8 . 8}$ \\
\hline Image-4 & $\mathbf{1 1 . 8}$ & $\mathbf{9 . 2}$ \\
\hline Image-5 & $\mathbf{1 2 . 0}$ & $\mathbf{9 . 2}$ \\
\hline
\end{tabular}

Table 4. RMSE results with noisy images of PSNR $=500$

\begin{tabular}{|c|c|c|}
\hline & $\begin{array}{c}\text { RMSE } \\
\text { Noisy Image }\end{array}$ & $\begin{array}{c}\text { RMSE } \\
\text { Denoised Image }\end{array}$ \\
\hline Image-1 & $\mathbf{3 . 2}$ & $\mathbf{2 . 9}$ \\
\hline Image-2 & $\mathbf{3 . 0}$ & $\mathbf{2 . 5}$ \\
\hline Image-3 & $\mathbf{3 . 0}$ & $\mathbf{2 . 3}$ \\
\hline Image-4 & $\mathbf{2 . 9}$ & $\mathbf{2 . 7}$ \\
\hline Image-5 & $\mathbf{3 . 0}$ & $\mathbf{2 . 3}$ \\
\hline
\end{tabular}

\section{CONCLUSION}

Proposed photon noise filtering techniques reduces photon noise significantly. Simulation results shows 20 to $50 \%$ reduction in RMSE. Count-wise SNR analysis is carried out. Significant SNR improvement SNR improvement is achieved. FFT analysis shows reduction of amplitude of high frequency noise components. Proposed technique is of low complexity and suitable for implementation. Simulation results can be further verified on more number of images before final implementation.

\section{ACKNOWLEDGMENT}

We gratefully acknowledge the constant encouragement and guidance received from Shri DRM Samudraiah- Prof. Satish Dhawan Scientist, Shri Saji A Kuriakose- DD-SEDA and Shri A. S. Kiran KumarDirector SAC. We are thankful to our colleagues of Payload Checkout Electronics Group for providing the images.

\section{REFERENCES}

[1] Abbas El Gamal, Helmy Eltoukhy, "CMOS image sensors", IEEE circuits and device magazine, May/June 2005, pp 6-20.

[2] M. Aharon, M. Elad, and A. Bruckstein. "K-SVD: An algorithm for designing overcomplete dictionaries for sparse representation" IEEE Trans. Signal Process., 54(11):4311\{ 4322, 2006.

[3] M. Collins, S. Dasgupta, and R. E. Schapire. "A generalization of principal components analysis to the exponential family” In NIPS, pages 617\{624, 2002.

[4] K. Dabov, A. Foi, V. Katkovnik, and K. O. Egiazarian, "Image denoising by sparse 3-D transform-domain 
collaborative filtering". IEEE Trans. Image Process., 16(8):2080\{ 2095, 2007.

[5] J. Mairal, F. Bach, J. Ponce, G. Sapiro, and A. Zisserman. "Non-local sparse models for image restoration". In ICCV, pages 2272\{2279, 2009.

[6] F. J. Anscombe, "The transformation of Poisson, binomial and negative binomial data," Biometrika, vol. 35, pp. 246-254, 1948.

[7] A. Foi. "Optimal inversion of the Anscombe transformation in low-count Poisson image denoising" IEEE Trans. Image Process., 20(1):99\{109, 2011.

[8] J. Boulanger, C. Kervrann, P. Bouthemy, P. Elbau, J-B. Sibarita, and J. Salamero. "Patch-based nonlocal functional for denoising uorescence microscopy image sequences" IEEE Trans. Med. Imag., 29(2):442\{454, 2010.

[9] B. Zhang, J. Fadili, and J-L. Starck. "Wavelets, ridgelets, and curvelets for Poisson noise removal". IEEE Trans. Image Process., 17(7):1093\{1108, 2008.

[10] J. S. Lee, "Digital Image Smoothing and the Sigma Filter", Computer Graphics Image Processing, Vol. 24, 1983, pp. 255-269.

[11] D. Van De Ville, M. Nachtagael, D. Van der Weken, E. E. Kerre, W. Philips, I. Lemahieu, "Noise Reduction by Fuzzy Image Filtering", IEEE Transactions on Fuzzy Systems, Vol. 11, No. 4, August, 2003, pp. 429-436.

[12] L. Alparone, S. Baronti, A. Garzelli, "A Hybrid Sigma Filter for Unbiased and Edge-Preserving Speckle Reduction", Proceedings of IEEE International Geoscience and Remote Sensing Symposium, IGARS '95, Vol. 2, 1995, pp. 1409-1411.

[13] Radu Ciprian Bilcu Markku Vehvilainen, "A Modified Sigma Filter for Noise Reduction in Images"

[14] R. C. Bilcu, M. Vehvilainen, "A New Method forNoise Estimation in Images", Proceedings of IEEE-EURASIP International Workshop on Nonlinear Signal and Image Processing, NSIP 2005, May 18-20, 2005, Sapporo, Japan, pp: 290-293.

[15] Ashok Kumar, Rajiv Kumaran, "Implementation of Multi Linear Gain Prior to image compression systems in Remote sensing electro optical payloads" in International Journal of Image, Graphics and Signal Processing, Peer review journal, Feb 2015, 3, pp: 51-57
End Electronics Division (Sensor Development Area). His research interest includes VLSI design, image processing, computation photography etc. He has been awarded "ISRO team excellence award-2009" for "Miniaturized camera electronics development". He has published 9 papers in various international/national conferences and peer reviewed journals. Contacts:

4396, SFED/SEG/SEDA, Space Applications Centre, Jodhpur Tekra, Ahmedabad-380015

Phone- 91-79-26914396

Email: ashokkumar@sac.isro.gov.in

Rajiv Kumaran, male, is a Scientist/Engineer at Space application Centre (ISRO). He passed his BE (E\&C) from GEC, Modasa. He joined Space Applications Center (SAC) in April 2000. Presently he is working on design and development of electronics for IRS payloads.

Contacts:

SFED / SEDA / Space Applications Center, Ahmedabad 380015

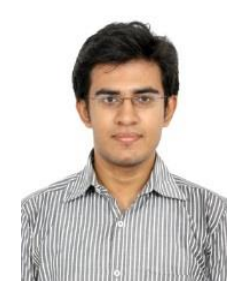

Harsh Trivedi, male, is currently pursuing his B.E. degree (Electronics and Communication) from L.D. College of Engineering, Ahmedabad (Gujarat Technological University). His major research interest area involves Image Processing, Computer Vision, Digital Signal Processing and VLSI. He has been honored by L. D. College of Engineering and Association of Consulting Civil Engineers (ACCE-India) for Outstanding Performance in the field of Electronics and communication during his study. He has also published a research paper in an international journal.

Contact:

Electronics and Communication Department, L. D. College of Engineering, Navrangpura, Ahmedabad-380015.

Phone: (+91)9409054362

Email: hctrivedi3@gmail.com

\section{Authors' Profiles}

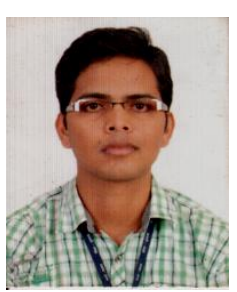

Ashok Kumar, male, is a Scientist/Engineer at Space application Centre (ISRO). He completed his BE degree (Electronics and communication) from University of Rajasthan in 2007. He is currently involved in advance research and development activities for future electro-optical payloads at Sensor Front

How to cite this paper: Ashok Kumar, Rajiv Kumaran, Harsh C Trivedi,"SNR Improvement by Photon Noise Filtering in Ocean Color Monitor Satellite Images", International Journal of Image, Graphics and Signal Processing(IJIGSP), Vol.8, No.2, pp.61-67, 2016.DOI: 10.5815/ijigsp.2016.02.08 